\title{
Co Orbitals View at a Glance
}

\author{
N.C. Pandey \\ Department of physics, Th. D. S. B. College Nainital, Kumaun University, Uttarakhand, India \\ Author: nawinpandeyphd@gmail.com \\ Available online at: www.isroset.org \\ Received: 03/Dec/2019, Accepted: 16/Dec/2019, Online: 31/Dec/2019
}

\begin{abstract}
Configuration for electrons of s2, p6, d10 \& f14 sub orbits is designed in view of the fact that each and every one electron of the atom can be issued to revolve in its own co orbital around the nucleus. The co orbitals view is determined by considering the nucleus at the point originating $\mathrm{O}$ of three mutually perpendicular specified axes $\mathrm{X}-\mathrm{X}^{\prime}, \mathrm{Y}^{-} \mathrm{Y}^{\prime}$ \& $\mathrm{Z}-\mathrm{Z}^{\prime}$. Coupled electrons of each sub orbit are given place to revolve around two mutually perpendicular co orbitals. Co orbitals of s2 sub orbit are surrounded two dimensionally, one by X Z' X' Z X enclosure while the other by Y Z Y' Z' Y enclosure respectively. The co orbitals of p6, d10 \& f14 sub orbits are spread over spherically around the nucleus. Such co orbitals are assisted by the rotation of $\mathrm{Z}-\mathrm{Z}^{\prime}, \mathrm{X}-\mathrm{X}^{\prime} \& \mathrm{Y}-\mathrm{Y}^{\prime}$ axes respectively towards various desired directions available around to revolve mutually in two perpendicular three dimensional planes.
\end{abstract}

Keywords : INSERT, FORMATTING, STYLE AND FONT (page and pictures, line spacing -1.08, special Intend - none and spacing before paragraph, headings- $1,2,4 \& 5$ bold, size $-11,10,9,10$ times new roman $\& 9$ times ,new roman)

\section{INTRODUCTION}

The general electronic structure of the atom assigned by $\mathrm{K}, \mathrm{L}, \mathrm{M}, \mathrm{N}, \mathrm{O}$ shells so called $\mathrm{n}=1,2,3$ \& 4 orbits are the main containers of electrons. Introduction of s2, p6, d10 \& f14 sub orbitals helped to redistribute electrons accordingly[1]. Because, Coulomb's law of electrostatic attraction between electron and the nucleus is responsible for giving revolutionary motion to the electron around the nucleus, such a motion of electron is used to find out the mechanism of coupling by dividing well known sub orbits into group of co orbitals[2]. This type of model of motion of electrons in allotted respective co orbitals does not allow electrons to collide with each other during the revolutionary motion of electrons. A comprehensive study is made regarding the presence of co orbitals and its application in finding the place for electrons revolving around the nucleus.

Thus, to assign the positions of all the electrons into the orbits of the atom, classification of sub orbits into co orbitals becomes very essential because of the fact that the electrons are strictly bound to revolve in two mutually different perpendicular co orbitals for coupling to take place in between a pair of two electrons. Now, it seems very essential to consider the theory of existence of co orbitals for viewing elections at a glance in its actual positions. The main characteristic motion of the electrons in various co orbitals of any sub orbit is based upon the following type of values of orbital and spin quantum numbers affiliated with the moving electrons.

1)The first type have the same orbital quantum number but have different spin quantum numbers.

2) The second type have the same spin quantum number but have different orbital quantum numbers.

Because, only two electrons of same orbital quantum number but having different spin quantum numbers can make a pair for coupling with each other, it becomes interesting to focus attention on this issue. It is newly investigated that such two electrons are found to revolve in two different co orbitals of same radius which are situated perpendicularly inclined on each other as in case of co orbitals designed for $1 \mathrm{~s} 2 \mathrm{sub}$ orbit. These conditions are treated to be thoroughly applicable while discussing the coupling mechanism as well as a place of co orbital for any of the electron in accordance to its orbital and spin quantum number.

\section{THEORY AND MECHANISM}

\section{[A] CO ORBITALS OF 1s2 SUB ORBIT}

Incase of first orbit $n=1,1=n-1=0$, the orbital quantum number has $21+1=1$ type of value only, hence both the electrons will have the same value of orbital quantum number[3]. The orbital quantum numbers of the coupling electrons can be denoted 
by $11=0 \& 11-=0$ respectively. Owing to take part in coupling, spin quantum number of these electrons are defined by $\mathrm{s}=+1 / 2$ $\& \mathrm{~s}=-1 / 2$. These two electrons are forced to revolve around by putting in two different but mutually perpendicular co orbitals nominated as 1 sl1 \&1sl1-. For the cause of coupling factor to take place in between this pair of electrons, the electron revolving in co orbital 1 sl1 couples with the electron revolving in 1sl1- co orbital.

Figure 1(A) shows that the coupling electrons have it's co orbitals respectively inclined by 90 degree from each other. At any time, these are moving in the same direction and are shown at positions $\mathrm{A}$ and $\mathrm{B}$ so that $\mathrm{OA}=\mathrm{OB}$. As, one electron goes from A to $A^{\prime}$, the second electron in the same time goes from $B$ to $B^{\prime}$, so that again $\mathrm{OA}^{\prime}=\mathrm{OB}^{\prime}$. Thus the distance of both the electrons remains the same at every time with respect to the nucleus situated at $\mathrm{O}$. It is there fore very familiar that both the electrons possess same sub orbit. Due to same radius of revolution around the same nucleus and having the same electronic mass under same value of centripetal force acting on both the electrons is of course due to the same velocity acquired by the both electrons [4]. It means to say that however, both the coupling electrons have the same radius and same velocity of revolution but the co orbitals for these are located mutually in two different circular paths, one enclosed by passing through $X$ $\mathrm{Z}^{\prime} \mathrm{X}^{\prime} \mathrm{Z}$ X axes around $\mathrm{O}$ while the other enclosed by passing through Y Z Y' Z' Y axes around $\mathrm{O}$.

\section{[B] CO ORBITALS OF 2p6 SUB ORBIT}

For $\mathrm{n}=2$, the maximum number of electrons is 8 and $\mathrm{l}=\mathrm{n}-1=1$. The orbital quantum number has $21+1=3$ types of values from +1 to -1 . Thus, eight electrons will have only three type of values of orbital quantum numbers $+1,0 \&-1$ but the radius of revolution of all co orbitals in both types of sub orbits $2 \mathrm{~s} 2 \& 2 \mathrm{p} 6$ will be the same so called the orbital radius of $\mathrm{L}$ shell. Firstly, the $2 \mathrm{~s} 2$ sub orbit is filled by 2 electrons. Both the electrons get the values $11=0 \& 11-=0$ for coupling with opposite spins. Two of the co orbitals are therefore $2 \mathrm{sl1}$ and 2sl1-. These co orbitals are situated at the height of radius of second orbit just above 1 sl1 \& 1sl1- co orbitals of 1s2 sub orbit with the same type of mechanism of revolution as shown in figure 1(B).

Figure 2(A) shows the spherical distribution of six co orbitals for the electrons of $2 \mathrm{p} 6$ sub orbit.The corresponding co orbitals are distinguished in two groups as exhibited in Figure 2(B). Three electrons of same $s=1 / 2$ spin quantum number but belong to different orbital quantum numbers as $0,+1 \&-1$ occupy positions of revolution in co orbitals termed as $2 \mathrm{pl} 1,2 \mathrm{pl} 2 \& 2 \mathrm{pl} 3$. The second group of remaining three electrons of opposite spin quantum number $\mathrm{s}=-1 / 2$ will have orbital quantum numbers 0 , $+1 \&-1$. The related co orbitals are 2pl1-, 2pl2- \& 2pl3-. The electron of first group lying in 2 pl1 co orbital couple with the electron of second group lying in 2pl1- co orbital by revolving mutually in two perpendicular co orbitals which are formulated around two mutually perpendicular rotational axes of Z-Z' axis. Thus electron of $2 \mathrm{pl} 1 \mathrm{co}$ orbital revolves around one rotational axis while the electron of 2pl1- co orbital revolves around another rotational axis. Both the rotational axes are situated in two entirely different but mutually perpendicular $3 \mathrm{~d}$ planes respectively. Rotation of Z-Z' axis towards two mutually perpendicular directions is used to provide co orbitals required for coupling between the appropriate pair of electrons. Co orbitals 2 pl1 \& $2 \mathrm{pl1}$ - are thus found to be situated at right angle to make couple. Co orbitals $2 \mathrm{pl} 2 \& 2 \mathrm{pl} 3$ of first group of electrons are associated with the electron of $2 \mathrm{pl} 1 \mathrm{co}$ orbital and are needed to be revolving around two different rotational axes of Z-Z' axis respectively. Such rotational axes must be $22.5^{\circ}$ angularly apart from the rotational axis selected by 2 pl1 co orbital. It can happen if and only if $2 \mathrm{pl} 1,2 \mathrm{pl} 2 \& 2 \mathrm{pl} 3$ co orbitals intersect each other at two points one situated at $\mathrm{Z}$ axis and another at $\mathrm{Z}^{\prime}$ axis. At these points, $2 \mathrm{pl} 2 \& 2 \mathrm{pl} 3$ co orbitals cross the middle co orbital $2 \mathrm{pl}$. However, the co orbitals of this group meet with each other at two points but all the three electrons pass one by one through these crossing points. Electron of $2 \mathrm{pl} 2$ having highest value of orbital quantum number leads while electron of $2 \mathrm{pl} 3$ having lowest value of orbital quantum number lags in motion with respect to the position of electron in 2 pl1 co orbital having zero value of orbital quantum number.

Similarly, the co orbitals 2pl2- \& 2pl3- of second group of electrons are associated with the electron of 2pl1- co orbital and are constructed to revolve respectively around two different rotational axes of $Z^{\prime}-Z$ axis which are $22.5^{\circ}$ angularly apart from the rotation axis that has been used in case of $2 \mathrm{pl1}$-. It can be only possible if $2 \mathrm{pl1-,} 2 \mathrm{pl2}-\& 2 \mathrm{pl3}$ - co orbitals pass through two common crossing points situated one at $Z^{\prime}$ axis and another at $Z$ axis . At these points 2pl2- \& 2pl3- cross the 2pl1- middle co orbital. Although, all the co orbitals of this group meet at two points but all the three electrons pass one by one through these crossing points. Electron of 2p12- having highest value of orbital quantum number leads while electron of 2p13- having lowest value of orbital quantum number lags in motion with respect to the position of electron of 2pl1- having zero value of orbital quantum number.

A clear configurational picture of co orbitals of $2 \mathrm{p} 6$ has been shorted out which shows that all the six co orbitals ate spread over spherically around the nucleus and intersect each other at two common points one situated in $\mathrm{Z}$ axis and another in $\mathrm{Z}^{\prime}$ axis. But, when the electrons of $+1 / 2$ spin quantum number moving in three dimensional circular co orbitals around the nucleus cross $\mathrm{Z}$ axis one by one, the electrons of $-1 / 2$ spin quantum number moving through another three dimensional circular co orbitals around the nucleus cross $Z^{\prime}$ axis one by one and vice versa. It can be manipulated that as electrons of 2 pl1 \& 2 pl1- co 
orbitals are found to be placed in two mutually perpendicular co orbitals for coupling with each other, the electrons of $2 \mathrm{pl} 2 \&$ 2 pl2- co orbitals are also found to be placed in two mutually perpendicular co orbitals for coupling with each other. Similarly electrons of $2 \mathrm{pl} 3 \& 2 \mathrm{pl} 3$ - co orbitals can also be found to be placed in two mutually perpendicular co orbitals for coupling to take place in between this pair of electrons also.

\section{[C] CO ORBITALS OF 3d10 SUB ORBIT}

For $\mathrm{n}=3$, the orbit may contain maximum 18 electrons. In order to isolate electrons, these are further divided into 3s 2 , 3p6 \& $3 \mathrm{~d} 10$ sub orbits. For third orbit $1=n-1=2$. All the 18 electrons will have $2 l+1=5$ types of values of orbital quantum numbers from +2 to -2 . But $3 \mathrm{~s} 2 \& 3 \mathrm{p} 6$ sub orbits will have the co orbital configuration of same type as attained by the co orbitals of $2 \mathrm{~s} 2 \& 2 \mathrm{p} 6$ sub orbits at the height of classical radius. The remaining ten electrons are the main constituents of $3 \mathrm{~d} 10$ sub orbit and have the values of orbital quantum numbers as $+2,+1,0,-1 \&-2$. The $3 \mathrm{~d} 10$ sub orbit is consisted of two groups of co orbitals. The first group contains co orbitals for five electrons each having spin quantum number $\mathrm{s}=+1 / 2$ and can be denoted as $3 \mathrm{dl} 1,3 \mathrm{dl} 2,3 \mathrm{dl} 3,3 \mathrm{dl} 4 \& 3 \mathrm{dl} 5$ where $11=0,12=+1,13=+2,14=-1 \& 15=-2$. The other group has co orbitals for remaining five electrons of opposite spin $\mathrm{s}=-1 / 2$ described as $3 \mathrm{dl} 1-, 3 \mathrm{dl} 2-, 3 \mathrm{dl} 3-, 3 \mathrm{~d} 14-\& 3 \mathrm{~d} 15-$ where $11-=0,12-=+1,13-=+2,14-=-1$ $\& 15-=-2$. These two groups of electrons revolve around the nucleus in mutually two perpendicular three dimensional planes around different rotational axes of $\mathrm{X}-\mathrm{X}$ ' in such a way that co orbitals of each group are angularly displaced by $15^{\circ}$ from its neighbouring co orbital. The middle co orbitals $3 \mathrm{dll} \& 3 \mathrm{dll}$ - of both the groups revolve around two mutually perpendicular rotational axes of X-X', hence couple with each other due to opposite spin $+1 / 2 \&-1 / 2$. Whether $3 \mathrm{~d} l 1$ or $3 \mathrm{~d} 11-$, each middle co orbital lies in between two leading and two lagging co orbitals.

Thus, it appears that all the ten co orbitals are spread over spherically around the nucleus by intersecting each other at two common crossing points one at $\mathrm{X}$ axis and the other at $\mathrm{X}^{\prime}$ axis. But when five electrons of same spin of a group cross the point at $\mathrm{X}$ axis one by one, the remaining five electrons of opposite spin of the second group cross the other point at $\mathrm{X}^{\prime}$ axis one by one and vice versa. Because, two such electrons can only couple with each other if and only if are found to be revolving across two mutually perpendicular co orbitals. The designing of co orbitals allow leading co orbitals $3 \mathrm{~d} l 2 \& 3 \mathrm{dl} 3$ associated with $3 \mathrm{dl} 1$ co orbital to couple with those leading co orbitals 3d12- \& 3dl3- associated with 3d11-co orbital respectively. Similarly, the lagging co orbitals $3 \mathrm{~d} 14 \& 3 \mathrm{~d} 15$ associated with $3 \mathrm{~d} 11$ co orbital couple with those lagging co orbitals 3dl4- \& 3dl5- associated with $3 \mathrm{~d} 11$ - co orbital respectively.

\section{[D] CO ORBITALS OF 4f14 SUB ORBIT}

Fourth orbit of $n=4$ consists maximum 32 electrons out of which 18 electrons are accommodated in various co orbitals of $4 \mathrm{~s} 2$, $4 \mathrm{p} 6 \& 4 \mathrm{~d} 10$ sub orbits. Because $1=\mathrm{n}-1=3$, all the electrons will have only $21+1=7$ types of values of orbital quantum numbers from +3 to -3 . Two electrons of $4 \mathrm{~s} 2$ sub orbit have only one type of value 0 . Six electrons of $4 \mathrm{p} 6$ sub orbit will have three types of values $+1,0 \&-1$. while ten electrons of $4 \mathrm{~d} 10$ sub orbit possess five types of values $+2,+1,0,-1 \&-2$. The coupling between any of the two electrons of $4 \mathrm{~s} 2$ or $4 \mathrm{p} 6$ or $4 \mathrm{~d} 10$ sub orbits will take place in a similar pattern as illustrated in the description of lower lying sub orbits of same kind. The only difference will be of radial height of the orbit from the nucleus.

The remaining 14 electrons are the main constituents of $4 \mathrm{f} 14$ sub orbit and have the values of orbital quantum numbers as +3 , $+2,+1,0,-1,-2 \&-3$. The $4 \mathrm{f} 14$ sub orbit is also consisted of two groups of co orbitals. The first group contains co orbitals for seven electrons each having spin quantum number $\mathrm{s}=+1 / 2$ and can be denoted by $4 \mathrm{fl} 1,4 \mathrm{fl} 2,4 \mathrm{fl} 3,4 \mathrm{fl} 4,4 \mathrm{fl} 5,4 \mathrm{fl} 6 \&$ $4 \mathrm{fl} 17$ where $11=0,12=+1,13=+2,14=+3,15=-1,16=-2 \& 17=-3$. The other group has co orbitals for seven electrons of opposite spin quantum number $\mathrm{s}=-1 / 2$ and are described as 4fl1-, 4fl2-, 4fl3-, 4f14-, 4f15-, 4fl6- \& 4f17- where 11- = 0, 12- = $+1,13-=+2,14-=+3,15-=-1,16-=-2 \& 17-=-3$. These two groups of electrons revolve around the nucleus in two mutually different perpendicular three dimensional planes around various rotational axes of $Y-Y^{\prime}$ in such a way that the co orbitals in each group are angularly displaced by $11.25^{\circ}$ apart from its neighbouring co orbital.

In this way, pairs of coupling electrons can be found to revolve around two mutually perpendicular co orbitals assisted by the rotation of $\mathrm{Y}^{-\mathrm{Y}^{\prime}}$ axis. The middle $4 \mathrm{fl} 1 \& 4 \mathrm{fl1}$ - co orbitals of each group revolve around two mutually perpendicular rotational axes of $Y-Y^{\prime}$ axis, hence couple due to having opposite spins $+1 / 2 \&-1 / 2$. Whether $4 f 11$ or $4 f 11-$, each middle co orbital lies in between three leading and three lagging co orbitals of electrons. At the points of crossing one at $Y$ axis and another at $Y^{\prime}$ axis, all the fourteen co orbitals intersect each other. But when seven electrons pass through touching $\mathrm{Y}$ axis one by one, the remaining seven electrons pass through touching $\mathrm{Y}^{\prime}$ axis one by one and vice versa. The leading co orbitals $4 \mathrm{fll} 2,4 \mathrm{fl} 3 \& 4 \mathrm{fl} 4$ associated with $4 \mathrm{fl} 1 \mathrm{co}$ orbital couple with those leading co orbitals $4 \mathrm{fl} 2-$, $4 \mathrm{fl} 3$ - \& 4fl4- associated with $4 \mathrm{fl} 1$ - co orbital respectively. In the same manner, lagging co orbitals $4 \mathrm{fl} 15,4 \mathrm{fl} 6 \& 4 \mathrm{fl} 7$ associated with $4 \mathrm{fl} 1$ co orbital coupe with those lagging co orbitals 4fl5-, 4fl6- \& 4fl7- associated with 4fl1- co orbital. 


\section{RESULTS AND DISCUSSION}

For studying the co orbitals of atom, it seems very necessary to keep the nucleus of atom at a point $\mathrm{O}$ through which three mutually perpendicular axes $\mathrm{X}-\mathrm{X}^{\prime}, \mathrm{Y}^{-\mathrm{Y}^{\prime}}$ \& $\mathrm{Z}-\mathrm{Z}^{\prime}$ pass. As, it is well known that an atom may have a bulk of many electrons, those electrons may require many co orbitals for revolution as electron in atom can not remain with out motion. There is a need of distribution of electrons in various orbits and then in sub orbits. The introduction of sub orbit gives an idea of carrying electrons towards various configurations. How and where these electrons are found to revolve is attained in this presented investigation by the concept of application of co orbitals view. Co orbitals are the actual users of electrons. Co orbital theory is based on the mechanism of coupling. According to this theory, only two electrons which have the same orbital quantum number and opposite spin quantum numbers can couple with each other. In addition, if these electrons are also found to revolve in two mutually perpendicular revolutionary co orbitals of same radius. This pattern of existence of co orbitals is thoroughly applied to find out the configuration of electrons around the nucleus.

The configuration of electrons of various sub orbits is prepared by the proper allotment of place for co orbitals for revolution of electrons around the nucleus. For this purpose, a circular co orbital made by the locus of motion of a point passing through $X Z^{\prime}$ $\mathrm{X}^{\prime} \mathrm{Z} \mathrm{X}$ axes in a two dimensional $\mathrm{X}-\mathrm{Z}$ plane around $\mathrm{O}$ is chosen for the revolutionary motion of one of the electron of $1 \mathrm{~s} 2 \mathrm{sub}$ orbit. The second electron of $1 \mathrm{~s} 2$ sub orbit revolves around another co orbital made by the locus of motion of another point passing through Y Z Y' Z' Y axes in Y-Z type of another two dimensional plane. Thus two separate co orbitals exist for 1s2 sub orbit, one revolving around $\mathrm{X} \mathrm{O} \mathrm{Z} \mathrm{plane} \mathrm{and} \mathrm{the} \mathrm{other} \mathrm{around} \mathrm{Y} \mathrm{O} \mathrm{Z} \mathrm{plane.} \mathrm{The} \mathrm{electrons} \mathrm{of} \mathrm{1s} 2$ sub orbit revolving in such two co orbitals possess the same value of orbital quantum number but opposite spin quantum numbers. In addition, these are situated mutually in perpendicular co orbital positions hence couple with each other. It is clear from the evaluated values of orbital quantum numbers for each of sub orbits s2, p6, d10 \& f14 that two electrons in each sub orbit have common zero value of orbital quantum number and are revolving in two such co orbitals in which instantaneous coordinates of electron can be easily evaluated. It should be assumed that at any instant of measurement when one electron of $1 \mathrm{~s} 2$ in co orbital $1 \mathrm{~s} 11$ passes by touching $\mathrm{X}$ axis at point $\mathrm{A}$, the second electron in its co orbital 1sl1- passes through the point $\mathrm{B}$ at $\mathrm{Y}$ axis. If r1 is the radius of first orbit, then the coordinates of $1 \mathrm{~s} 2$ electrons will be one in $\mathrm{X}$ axis at $\mathrm{r} 1,0,0$ and the other in $\mathrm{Y}$ axis at 0 , r1, 0 .

Similarly, if $r 2$ is the radius of $n=2$ orbit, the pair electrons of $2 \mathrm{~s} 2$ will be observed one at $\mathrm{X}$ axis in 2 sl1 co orbital having coordinates $\mathrm{r} 2,0,0$ and the other at $\mathrm{Y}$ axis in $2 \mathrm{~s} 11$ - co orbital having coordinates $0, \mathrm{r} 2,0$. Thus co orbitals of $2 \mathrm{~s} 2$ sub orbit will find its positions just above the positions of co orbitals of $1 \mathrm{~s} 2$ sub orbit. In case of treatment of co orbitals of 2 p6 sub orbit, coupling pairs of co orbitals are assisted by the rotation of $Z-Z^{\prime}$ axis towards two mutually perpendicular directions. The motion of electrons is selected in such way that both electrons of the coupling pair must revolve around the nucleus in two different co orbitals appearing mutually perpendicular. Two coupling electrons having zero value of orbital quantum number are situated in co orbitals 2 pl1 \& 2pl1-, each of orbital radius $r 2$. If the electron of 2 pl1 is at $Z$ axis revolving via three dimensional $Z^{\prime} X^{\prime} Y^{\prime}, Z^{\prime}$ $X^{\prime} Y^{\prime}, Z^{\prime} X$ Y \& Z X Y planes, the electron of 2pl1- is at $Z^{\prime}$ axis revolving via another three dimensional $Z^{\prime} X^{\prime} Y, Z^{\prime} Y, Z^{\prime}$ $Y^{\prime} \& Z^{\prime} X Y^{\prime}$ planes. The instantaneous coordinates of electron of 2 pl1 when at $Z$ axis is therefore $0,0, r 2$ while that of $2 p 11-$ when at $Z^{\prime}$ axis is $0,0,-r 2$. It should be remembered that eight electrons of $2 \mathrm{~s} 2 \& 2 \mathrm{p} 6$ have same size of co orbitals. All the eight co orbitals intersect each other at two common points one situated at $\mathrm{Z}$ axis while the other at Z' axis. But, 2 s2 electrons revolve entirely in two dimensional planes while the electrons of 2 p6 revolve through out in three dimensional planes. Thus the path for every electron is chosen absolutely separate. When the electrons of each group of $2 \mathrm{p} 6$ have left the positions from $Z$ and $Z^{\prime}$ axes one by one, then the electrons of 2 s 2 enter at these two points on $Z^{\prime}$ and $Z$ axes one by one successively.

For $n=3$, the co orbitals of $3 \mathrm{~s} 2$ will be found at the height $r 3$ of radius of third orbit just above the co orbitals of $2 \mathrm{~s} 2$. The co orbitals can be written as 3sl1 \& 3sl1- while the instantaneous coordinates of corresponding electrons should be $\mathrm{r} 3$, 0 , 0 at $\mathrm{X}$ axis and $0, \mathrm{r} 3,0$ at $\mathrm{Y}$ axis. The appropriate pair of electrons of $3 \mathrm{p} 6$ sub orbit couple with matching co orbitals just above of the co orbitals of 2 p6 sub orbit. The main two electrons in 3pl1 and 3pl1-co orbitals which have zero value of orbital quantum number are coordinated by $0,0, \mathrm{r} 3$ and $0,0,-\mathrm{r} 3$ respectively. The electrons of $3 \mathrm{~d} 10$ sub orbit are supposed to revolve in two groups of five co orbitals, each co orbital revolves around different rotational axis of X-X'. The main electrons which have zero value of orbital quantum number and couple due to opposite spins and are found in mutually two perpendicular co orbitals $3 \mathrm{~d} l 1$ and 3d11-. During motion, the electron of 3dl1 co orbital touches both $X$ and $X^{\prime}$ axes one by one and revolves around the rotational axis of $\mathrm{X}-\mathrm{X}^{\prime}$ axis via $\mathrm{X} Y \mathrm{Z}, \mathrm{X}^{\prime} \mathrm{Y} \mathrm{Z}, \mathrm{X}^{\prime} \mathrm{Y}^{\prime} \mathrm{Z}^{\prime} \& \mathrm{X} \mathrm{Y}^{\prime} \mathrm{Z}^{\prime}$ planes. The instantaneous position of electron in $3 \mathrm{~d} l 1$ co orbital is taken when it is in between $\mathrm{Y}$ and $\mathrm{Z}$ axes at an angle of $45^{\circ}$ from each $\mathrm{O}-\mathrm{Y}$ and $\mathrm{O}-\mathrm{Z}$ axes, hence will have the coordinates $0, \mathrm{r} 3 / \sqrt{ } 2, \mathrm{r} 3 / \sqrt{ } 2$. The electron of $3 \mathrm{~d} 11$ - co orbital moves by touching $X^{\prime} \& X$ axes one by one and revolves around another rotational axis of $\mathrm{X}^{\prime}-\mathrm{X}$ via $\mathrm{X}^{\prime} \mathrm{Y}^{\prime} \mathrm{Z}, \mathrm{X} \mathrm{Y}^{\prime} \mathrm{Z}, \mathrm{X} \mathrm{Y} \mathrm{Z}^{\prime} \& \mathrm{X}^{\prime} \mathrm{Y} \mathrm{Z}^{\prime}$ planes. The instantaneous position of electron in $3 \mathrm{~d} l 1$ - co orbital is used when it is in between $\mathrm{Y}^{\prime}$ and $\mathrm{Z}$ axes at an angle of $45^{\circ}$ from each $\mathrm{O}-\mathrm{Y}^{\prime}$ and $\mathrm{O}-\mathrm{Z}$ axes having coordinates 0 , $\mathrm{r} 3 / \sqrt{ } 2, \mathrm{r} 3 / \sqrt{ } 2$. 
Now, let us find out the main coordinates of the electrons of fourth orbit $n=4$ in co orbitals of all sub orbits $4 \mathrm{~s} 2,4 \mathrm{p} 6,4 \mathrm{~d} 10$ \& $4 \mathrm{f} 14$ all of same radius $\mathrm{r} 4$. The $4 \mathrm{~s} 11$ and $4 \mathrm{~s} 11$ - co orbitals of $4 \mathrm{~s} 2$ sub orbit find positions according to the same pattern as lower lying co orbitals of $1 \mathrm{~s} 2,2 \mathrm{~s} 2 \& 3 \mathrm{~s} 2 \mathrm{sub}$ orbits get. The instantaneous coordinates of the two such electrons are r4, 0,0 and 0 , r 4 , 0 . The co orbitals of $4 \mathrm{p} 6$ sub orbit are also filled in step wise as lower lying $2 \mathrm{p} 6$ and $3 \mathrm{p} 6$ sub orbits constitute co orbitals. The main two electrons of $4 \mathrm{pl} 1$ and $4 \mathrm{pl1}$ - co orbitals are instantaneously designated by the coordinates 0 , 0 , r4 and 0 , 0 , -r 4 respectively. For coupling of electrons of $4 \mathrm{~d} 10 \mathrm{sub}$ orbit, the presence of mutually perpendicular co orbitals is very similar to that of co orbitals of $3 \mathrm{~d} 10 \mathrm{sub}$ orbit. The only difference is of radius of co orbitals. The main two electrons which have zero value of orbital quantum number and which couple with each other due to opposite spins revolve around nucleus in two perpendicular co orbitals $4 \mathrm{dl} 1$ and $4 \mathrm{~d} 11$ - co orbitals. The co orbitals view of these two electrons will be observed by coordinates situated at $0, \mathrm{r} 4 / \sqrt{ } 2, \mathrm{r} 4 / \sqrt{ } 2$ and $0,-\mathrm{r} 4 / \sqrt{ } 2, \mathrm{r} 4 / \sqrt{ } 2$ respectively.

Co orbitals of $4 \mathrm{f} 14$ sub orbit can be observed to exist in two groups. Each co orbital of the group is present around different axis. These axes are situated towards various angles of rotation of $\mathrm{Y}-\mathrm{Y}^{\prime}$ axis. The middle co orbitals of both the group of co orbitals have zero value of orbital quantum number and are denoted by $4 \mathrm{fl} 1$ and $4 \mathrm{fl} 1-$. One electron of the coupling pair is suitable to revolve around one rotational axis of $\mathrm{Y}^{-} \mathrm{Y}^{\prime}$ in $4 \mathrm{fl} 1$ co orbital by touching $\mathrm{Y}$ and $\mathrm{Y}^{\prime}$ axes one by one during motion in three dimensional $\mathrm{Y} \mathrm{Z}^{\prime} \mathrm{X}^{\prime}, \mathrm{Y}^{\prime} \mathrm{Z}^{\prime} \mathrm{X}^{\prime}, \mathrm{Y}^{\prime} \mathrm{ZX}$ \& Y Z X planes while the second electron of the coupling pair is found to revolve around another rotational axis of $Y^{\prime}-Y$ axis in $4 \mathrm{fl} 1$ - co orbital by touching $\mathrm{Y}^{\prime}$ and $\mathrm{Y}$ axes one by one during motion in three dimensional $\mathrm{Y}^{\prime} \mathrm{Z}^{\prime} \mathrm{X}, \mathrm{Y} \mathrm{Z}^{\prime} \mathrm{X}, \mathrm{Y} \mathrm{Z} \mathrm{X}^{\prime}$ \& $\mathrm{Y}^{\prime} \mathrm{Z} \mathrm{X}^{\prime}$ planes. But the instantaneous position of electron in $4 \mathrm{fl1}$ is chosen in between $Z^{\prime}$ and $X^{\prime}$ axes at an angle of $45^{\circ}$ from each $O-X^{\prime}$ and $O-Z^{\prime}$ axes depicted by the coordinates $-\mathrm{r} 4 / \sqrt{2}$, 0 , -r $4 / \sqrt{2}$ while the position of electron in $4 \mathrm{fl} 1$ - lies in between $\mathrm{Z}^{\prime}$ and $\mathrm{X}$ axes at an angle of $45^{\circ}$ from each $\mathrm{O}-\mathrm{X}$ and $\mathrm{O}-\mathrm{Z}^{\prime}$ axes depicted by the coordinates $\mathrm{r} 4 / \sqrt{ } 2,0,-\mathrm{r} 4 / \sqrt{ } 2$. The instantaneous coordinates of all most of the electrons which have zero value of orbital quantum number have been so determined to get the co orbitals view. At any instant of time, the points through which the electrons are viewed to pass during the revolution in its co orbitals around the nucleus is termed as the co orbitals view at a glance.

\section{CONCLUSION}

The theory of existence of co orbitals presented here is based upon the mechanism of coupling between two electrons of same orbital but having opposite spin quantum numbers. Revolution of such electrons in two separate and mutually perpendicular co orbitals of same radius is helpful to know the actual configuration of electrons in a very little space around the nucleus. The co orbital path available to follow by each of the coupling electron is only understood in this way. Therefore such a pattern is very useful in finding co orbitals for electrons following a disciplinary law of motion around the nucleus responsible for the permanent and necessary establishment of atomic stability.

\section{ACKNOWLEDGEMENT}

The author is very kind full to his guide Dr. J. C. Joshi for his continuous support to deal with affiliated matter in hand. The author likes to dedicate this research paper in memory of Dr. D. D. Pant.

\section{REFERENCES}

[1] D.P. Khandelwal “OPTICS AND ATOMIC PHYSICS”, Himalaya Publication, India, pp. 309 -313, 2008.

[2] J.P. Agarwal "FUNDAMENTALS OF MODERN PHYSICS", Pragati Publication, India, pp. 87, 2013.

[3] S.L. Gupta “ATOMIC AND MOLECULAR PHYSICS”, Pragati Publication, India, pp. 5-8, 1989.

[4] R. Kumar “ATOMIC \& MOLECULAR SPECTRA : LASER”, Ramnath Publication, India, pp. 8, 2017. 


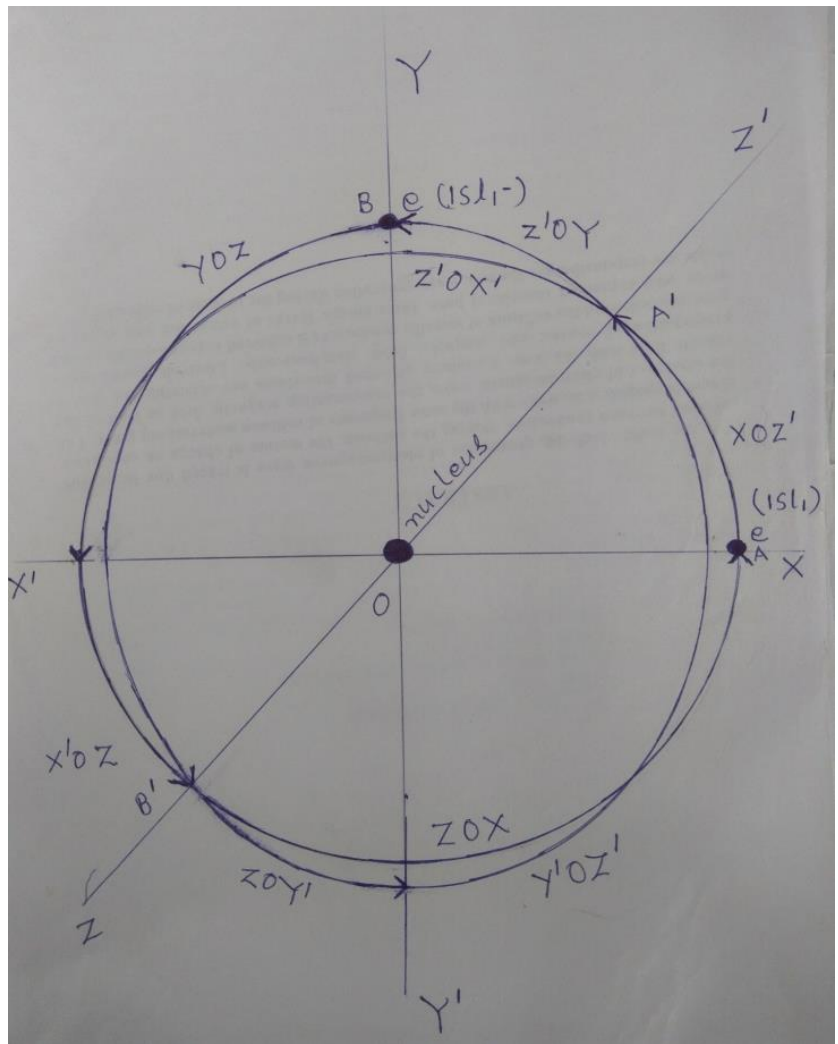

Figure 1(A).Two dimensional enclosures view of co orbitals of 1s2 sub orbit.

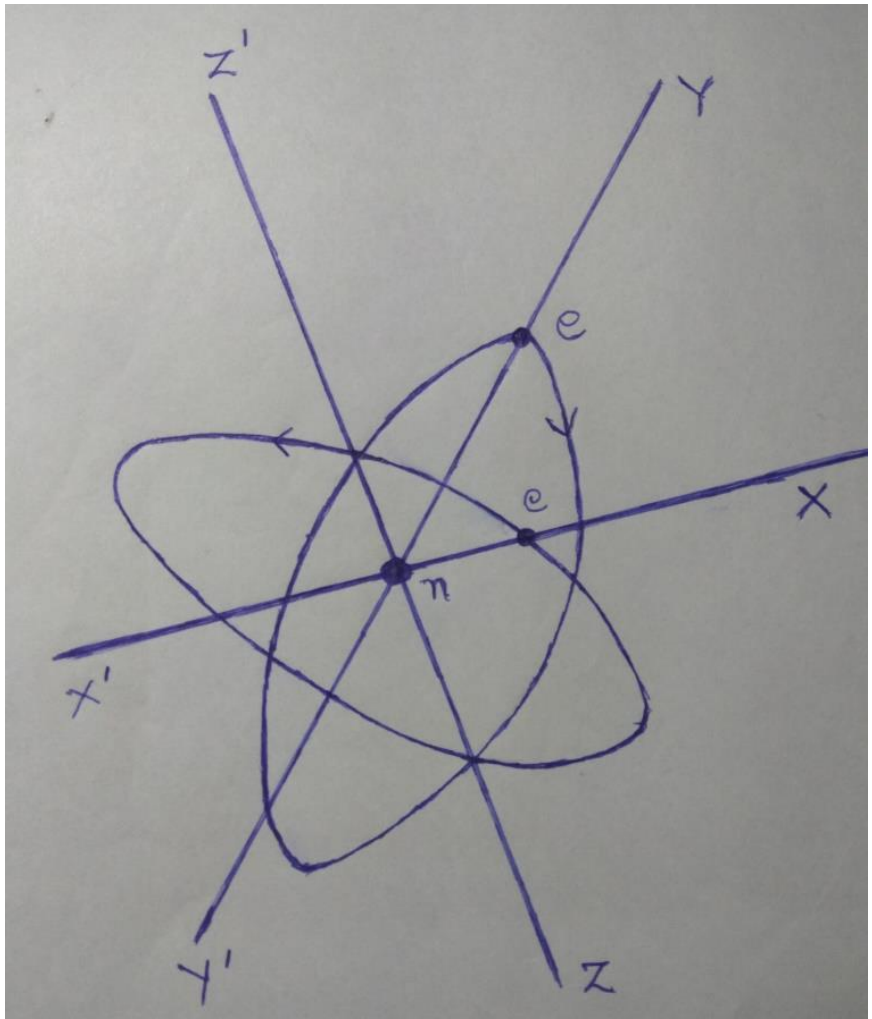

Figure 1(B). Co orbitals view of 1s2 sub orbit. 


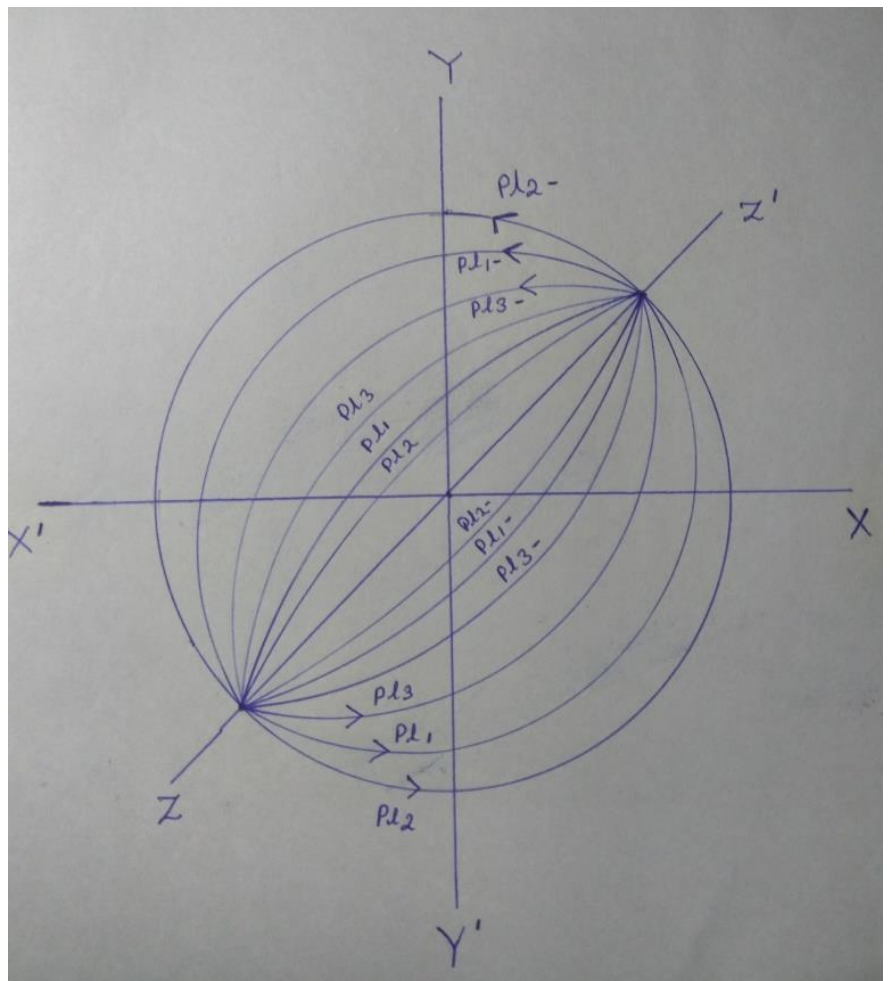

Figure 2(A). Spherical distribution of co orbitals of $2 p 6$ sub orbit.

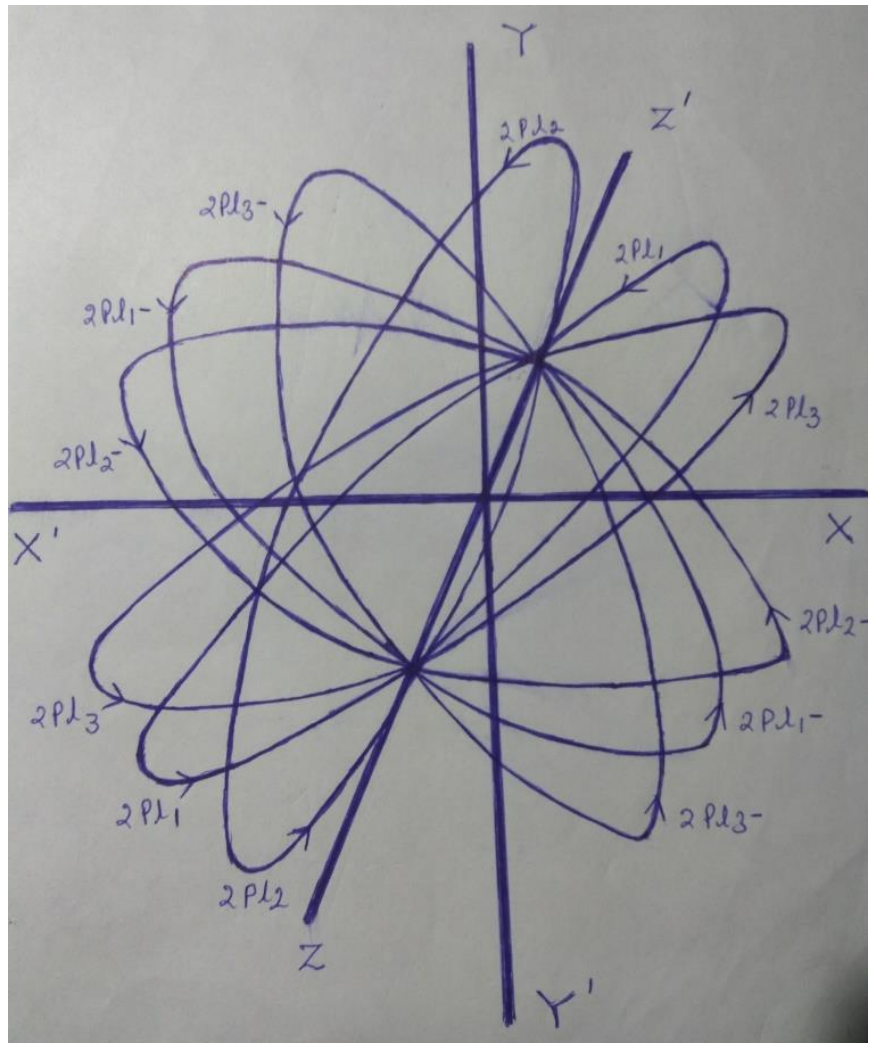

Figure 2(A). Spreading of co orbitals of $2 p 6$ sub orbit as a result of intersection at two points on Z-Z' axis. 